\title{
Transtornos Mentais Menores entre Estudantes de Medicina
}

\author{
Minor Mental Disorders Among Medical \\ Students
}

Emmanuelle Santana Rocha ${ }^{I}$ André Petraglia Sassi ${ }^{1}$

\section{PALAVRAS-CHAVE \\ - Transtornos Mentais. \\ - Estudantes de Medicina. \\ - Fatores de Risco. \\ - Saúde Mental.}

Recebido em: 25/11/2012

Reencaminhado em: 19/03/2013

Aprovado em: 19/03/2013

REVISTA BRASILEIRA DE EDUCAÇÃO MÉDICA

\section{RESUMO}

Objetivo: O objetivo do estudo foi estimar a prevalência de Transtornos Mentais Menores (TMM) entre os estudantes do curso de Medicina da Universidade Federal da Paraíba (UFPB) e avaliar possíveis correlações entre TMM e fatores de risco. Métodos: Estudo transversal realizado de abril a agosto de 2012 com 384 alunos do curso de Medicina. O questionário utilizado foi autoaplicável e anônimo. Foram coletados dados sociodemográficos e a rede de apoio social. Para o rastreamento de TMM, utilizou-se o Self-Reporting Questionnaire (SRQ-20). Resultados: A prevalência total de TMM encontrada foi de 33,6\%, que esteve independentemente associada ao período do curso ( $p<$ 0,$001 ; 9,7 \%$ a 63,3\%), à idade ( $p<0,05 ; 25 \%$ a 42,6\%), a não seguir uma religião ( $p<0,05 ; 44,8 \%$ ). Conclusão: Os dados demonstram elevada prevalência de TMM nessa população e a importância de subsidiar ações para prevenção e cuidado com a saúde mental dos estudantes de Medicina, melhorando sua qualidade de vida.

\section{ABSTRACT}

Objective: The objective of this study was to estimate the prevalence of minor mental disorders (MMR) among medical students of the Federal University of Paraíba (UFPB) and to evaluate possible correlations between MMR and risk factors. Methods: Cross-sectional study conducted from April to August 2012 involving 384 medicine students. The questionnaire was self-administered and anonymous. We collected sociodemographic data and information on their social support networks. For screening MMR, we used the Self-Reporting Questionnaire (SRQ-20). Results: The overall prevalence of TMM was found to be $33.6 \%$, which was independently correlated to the duration of the course ( $p<0.001,9.7 \%$ to $63.3 \%$ ), age ( $p<0.05,25 \%$ to $42.6 \%$ ), not following a religion ( $p<0.05$, $44.8 \%)$. Conclusion: The data demonstrate a high prevalence of $M M R$ in this population group and the importance of support programs for mental health prevention and care of medical students, improving their quality of life. 


\section{INTRODUÇÃO}

Transtornos mentais menores (TMM) representam quadros menos graves e mais frequentes de transtornos mentais. Os sintomas incluem alterações de memória, dificuldade de concentração e de tomada de decisões, insônia, irritabilidade e fadiga, assim como queixas somáticas (cefaleia, falta de apetite, tremores, sintomas gastrointestinais, entre outros) ${ }^{1}$.

Ser portador de TMM representa custos em termos de sofrimento psíquico e impacto nos relacionamentos e na qualidade de vida, comprometendo o desempenho nas atividades diárias, sendo substrato para o desenvolvimento de transtornos mais graves ${ }^{1}$.

A epidemiologia define como fatores de risco variáveis sociais ou biológicas que, quando presentes, aumentam a probabilidade de ocorrência de dada doença. Nesse sentido, a literatura tem apontado algumas características que, quando presentes, se associam ao risco aumentado de morbidade mental menor ${ }^{2}$.

Os transtornos mentais têm maior chance de surgir pela primeira vez no início da vida adulta, principalmente no período universitário ${ }^{3,4}$. As situações de perda presentes no desenvolvimento normal se acentuam quando os jovens ingressam na universidade, pois se afastam de um círculo conhecido de relacionamentos familiares e sociais, o que pode desencadear situações de crise $^{3,5}$. Tem-se encontrado maior taxa de sofrimento mental entre estudantes universitários, se comparados com jovens da mesma idade que não estão na universidade ${ }^{6}$.

A prevalência de TMM é mais elevada entre os estudantes de Medicina do que na população geral, pois eles estão sujeitos a potentes estressores, como uma rede de apoio deficiente, sobrecarga de conhecimentos, competição no processo de seleção, dificuldade na administração do tempo, individualismo, responsabilidade e expectativas sociais do papel de médico, contato com a morte e processos patológicos, o exame físico de pacientes, o medo de adquirir doenças, o medo de cometer erros e sentimentos de impotência diante de certas doenças. Esses fatores podem levar os estudantes a acionar mecanismos de defesa psicológicos, tais como dissociação ou isolamento afetivo ${ }^{1}$.

Alguns estudos demonstram que o gênero feminino é a variável mais intensamente associada de modo independente a ter transtorno psiquiátrico menor ${ }^{7,8}$.

Os estudantes com TMM geralmente apresentam pior relacionamento com amigos, sentem-se discriminados e não apoiados pelos pais, têm problemas de uso de álcool ou drogas na família e provêm, em maior número, de ensino fundamental realizado em escola pública ${ }^{3}$.

No Brasil, em 1958, Loreto ${ }^{9}$ realizou o primeiro estudo sobre saúde mental em estudantes universitários. Identificou que cerca de um terço dos estudantes atendidos no Serviço de Higiene Mental para Estudantes da Universidade Federal de Pernambuco (UFPE) apresentava sintomatologia neurótica, e dois terços, dificuldades de personalidade e padrões de reações emocionais inadequados. As queixas se relacionaram mais à vida pessoal do que à acadêmica, apesar de os estudantes reconhecerem que as dificuldades emocionais prejudicavam o rendimento nos estudos ${ }^{9,10}$.

Estudo na Universidade Federal de Santa Maria, usando o Self-Reporting Questionnaire (SRQ-20) mostrou que a prevalência de TMM entre os estudantes do primeiro ao décimo semestre do curso de Medicina foi de 31,7\%. Mostrou também haver aumento progressivo nos escores de depressão dos estudantes durante a escola médica, o que sugere que o sofrimento mental para essa população é crônico e persistente ${ }^{11}$.

Facundes e Ludermir ${ }^{12}$ encontraram na UFPE a prevalência de $34,1 \%$ de transtornos mentais menores, sendo significativamente maior entre os que se sentiam sobrecarregados e os que afirmaram a presença de situações especiais durante a infância e adolescência.

Pereira Lima et al. ${ }^{13}$, na Faculdade de Medicina de Botucatu, encontraram prevalência de 44,7\% de TMM, associando-se independentemente a dificuldade para fazer amigos, avaliação ruim sobre desempenho escolar, pensar em abandonar o curso e não receber o apoio emocional necessário.

Em estudo realizado com estudantes do curso de Medicina da Universidade Federal do Espírito Santo ${ }^{1}$ para avaliar possíveis correlações entre TMM e fatores de risco, a prevalência total de TMM encontrada foi de 37,1\%, associada a não receber apoio emocional necessário.

No Brasil há escassez de estudos epidemiológicos sobre a morbidade psiquiátrica em estudantes universitários. A grande maioria dos estudos nacionais publicada está relacionada aos levantamentos dos índices de utilização dos serviços de saúde mental oferecidos pelas universidades, como no caso da clínica-escola de Psicologia da Universidade São Francisco, São Paulo ${ }^{14}$

O estudo visa identificar a prevalência de transtornos mentais menores entre estudantes do curso de Medicina da UFPB e determinar a correlação com possíveis fatores de risco.

\section{MÉTODOS}

\section{Modelo e Local de Estudo}

Estudo transversal, realizado entre abril e agosto de 2012, com 384 alunos do primeiro ao sexto ano do curso de Medicina da UFPB, selecionados de acordo com a presença em sala no momento da pesquisa. 


\section{Seleção da Amostra e Coleta de Dados}

O curso de Medicina da UFPB tem 643 alunos regularmente matriculados no semestre 2012.1, com 53 (+/- 7) alunos em cada período da graduação, do $1^{\circ}$ ao $12^{\circ}$.

Foram selecionados 32 alunos por turma, entregando-se os questionários àqueles que estavam presentes na aula no momento da pesquisa; os que estavam no internato ( $9^{\circ}$ ao $12^{\circ}$ ) foram abordados no intervalo de suas atividades.

O tamanho da amostra foi calculado para estimar a taxa de prevalência de TMM em estudantes de Medicina da UFPB, com um intervalo de confiança de $95 \%$. Tomou-se como base para cálculo uma taxa média de prevalência de $35 \%{ }^{15}$, com variação de $5 \%$. Considerando uma possível perda de aproximadamente $20 \%$, o tamanho da amostra final necessário foi de 384 estudantes.

\section{Instrumento de Coleta}

Os questionários utilizados na pesquisa foram autoaplicáveis e anônimos.

O questionário sociodemográfico e psicossocial é baseado nas informações coletadas em estudos anteriores ${ }^{1}$ (p. 19). Esse instrumento contém dados socioeconômicos (período, idade, sexo, cor, religião, renda), sobre a rede de apoio (relacionamento dos pais, grupo de amigos, rejeição, apoio emocional) e história de patologias psiquiátricas (história familiar de TMM ou de doença mental, tratamento psiquiátrico medicamentoso, tratamento psicoterapêutico).

Para o rastreamento de TMM, foi utilizado o SRQ-20, instrumento desenvolvido para rastrear distúrbios psiquiátricos em centros de atenção primária à saúde, validado no Brasil e recomendado pela Organização Mundial da Saúde. Esse instrumento é composto por 20 perguntas com respostas "sim" ou "não", sendo quatro perguntas sobre sintomas físicos e 16 sobre desordens psicoemocionais. A cada resposta "sim" é atribuído um ponto, resultando numa pontuação final que varia de 0 a 20 pontos.

Os alunos foram distribuídos em dois grupos de acordo com a pontuação no SRQ-20, pois o ponto de corte é diferente. Homens com pontuação inferior ou igual a cinco e mulheres com pontuação inferior ou igual a sete foram classificados como "não suspeitos" para TMM. Homens com pontuação superior ou igual a seis e mulheres com pontuação superior ou igual a oito foram classificados como "suspeitos" para TMM.

Os alunos foram contatados em sala de aula, na universidade, ou no serviço em que prestavam seu rodízio de internato; todos leram e assinaram o termo de consentimento e receberam os questionários para serem respondidos. $\mathrm{O}$ aluno que não respondeu ao questionário foi considerado como "perda".

\section{ANÁLISE DE DADOS}

Foi utilizado o programa IBM SPSS Statistics 20.0. Foi criada uma variável TMM a partir do escore total de cada indivíduo no SRQ-20, no qual os sujeitos foram classificados como "suspeitos" de TMM ou não, de acordo com os pontos de corte.

Inicialmente, foi realizada uma análise descritiva da população estudada segundo as variáveis em estudo (presença de TMM e variáveis sociodemográficas).

Para análise dos dados, as variáveis categóricas foram representadas por suas frequências absolutas. Ao considerar a variável de desfecho (presença ou ausência de TMM) e as possíveis exposições (presença ou ausência de cada fator), a análise bivariada trabalhou com uma resposta do tipo dicotômica com comparação de dois grupos.

O teste estatístico utilizado foi o qui-quadrado. Serão consideradas variáveis estatisticamente significativas aquelas com $\mathrm{p}<0,05$.

\section{RESULTADOS}

Dentre os 384 questionários entregues, obteve-se retorno de 354 (92,2\% da amostra selecionada, com perda de 7,8\%, o que não foi significativo para o estudo), representados por 118 $(33,33 \%)$ alunos do $1^{\text {o }}$ ao $4^{\text {o }}$ período, $140(39,54 \%)$ do $5^{\text {o }}$ ao $8^{\text {o }}$ período e $96(27,11 \%)$ do $9^{\circ}$ ao $12^{\circ}$ período. Quanto à idade, 54 $(15,3 \%)$ apresentavam até 19 anos, $172(48,6 \%)$ tinham de 20 a 23 anos e 128 (36,2\%) estavam com 24 anos ou mais. Quanto ao gênero, 175 (49,4\%) eram do sexo feminino e 179 (50,4\%) do sexo masculino. Em relação à cor, 193 (54,5\%) se consideravam da cor branca, 138 (39) da cor parda, 13 (3,7\%) da cor preta, 4 $(1,1 \%)$ da cor amarela, $3(0,8 \%)$ indígena e $3(0,8 \%)$ não informaram a cor. Quanto à renda familiar, $27(7,6 \%)$ possuíam renda de até R\$1.500, 209 (59\%) de R\$ 1.500 a 10.000, 91 (25,7\%) renda maior que $\mathrm{R} \$ 10.000$ e 27 (7,6\%) não informaram.

Em relação à saúde mental, 119 estudantes $(33,6 \%)$ obtiveram pontuação que os classifica como casos "suspeitos" de TMM (Gráfico 1). O estudo apontou maior prevalência de TMM entre os alunos do curso básico (41,5\%) e do clínico $(42 \%)$, sendo menor no internato $(18,5 \%)(p<0,001)$. Estratificando os resultados, notou-se que a maior prevalência foi encontrada no quinto período $(63,3 \%)$ e a menor prevalência foi encontrada no décimo período, com apenas $9,7 \%$.

Outras variáveis estatisticamente significativas relacionadas ao TMM (Tabela 1) foram: idade menor que 19 anos, com $42,6 \%$ ( $\mathrm{p}=0,027)$; não seguir alguma religião, com $44,8 \%$ ( $\mathrm{p}=$ $0,005)$; dificuldade de fazer amigos, $77,5 \%(p=0,005)$; sentir-se rejeitado por amigos ou outros na mesma faixa etária, $82,9 \%$ (p $<0,001)$; sentir que não recebe o apoio emocional que necessita, $52,9 \%(\mathrm{p}<0,001)$; e ter história familiar de doença psiquiátrica, com $43,8 \%(p=0,003)$. 


\section{GRÁFICO 1}

Distribuição entre períodos de estudantes "suspeitos" e "não suspeitos" de transtornos mentais menores (TMM) do curso de Medicina da UFPB, 2012 ( $\mathrm{N}=354)$

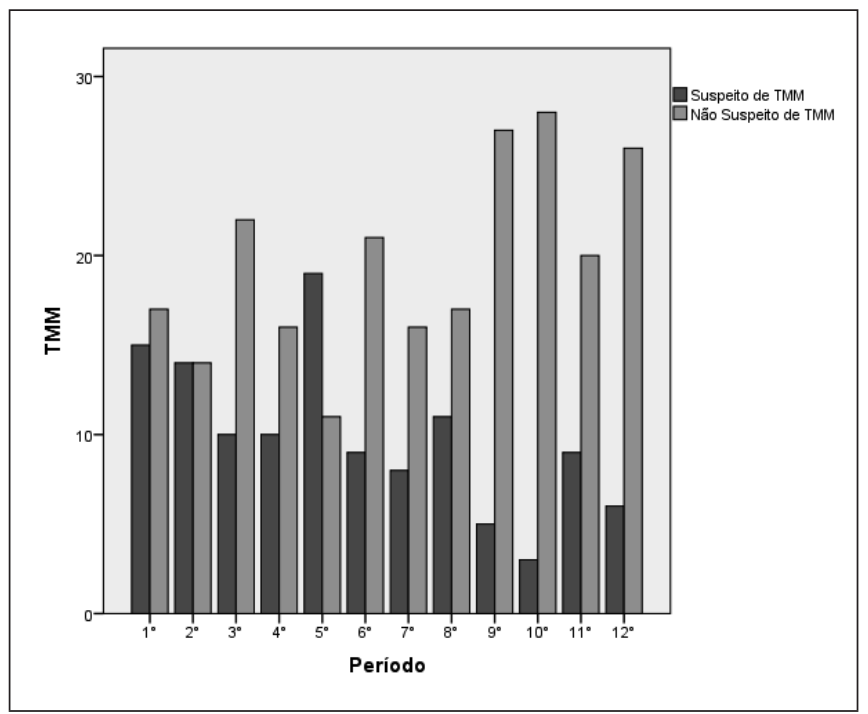

TABELA 1

Variáveis estatisticamente significativas associadas com casos suspeitos de transtornos mentais menores (TMM) entre estudantes de Medicina da UFPB, $2012(\mathrm{~N}=354)$

\begin{tabular}{|c|c|c|c|c|}
\hline Variáveis & $\mathbf{N}$ & $\%$ & $\%$ TMM & $\mathbf{P}$ \\
\hline Idade & & & & 0,027 \\
\hline Até 19 anos & 54 & 15,25 & 42,6 & \\
\hline 20 a 23 anos & 172 & 48,6 & 37,2 & \\
\hline 24 anos ou mais & 128 & 36,15 & 25 & \\
\hline Segue alguma religião & & & & 0,007 \\
\hline Sim & 258 & 72,88 & 29,5 & \\
\hline Não & 96 & 27,11 & 44,8 & \\
\hline $\begin{array}{l}\text { Sente dificuldade de fazer } \\
\text { amigos }\end{array}$ & & & & $<0,001$ \\
\hline Sim & 40 & 11,3 & 77,5 & \\
\hline Não & 314 & 88,7 & 28 & \\
\hline $\begin{array}{l}\text { Sente-se rejeitado por amigos } \\
\text { ou outros da mesma idade }\end{array}$ & & & & $<0,001$ \\
\hline Sim & 41 & 11,6 & 82,9 & \\
\hline Não & 313 & 88,4 & 27,2 & \\
\hline $\begin{array}{l}\text { Sente que recebe o apoio } \\
\text { emocional necessário }\end{array}$ & & & & $<0,001$ \\
\hline Sim & 268 & 75,7 & 27,2 & \\
\hline Não & 85 & 24 & 52,9 & \\
\hline $\begin{array}{l}\text { Tem história familiar de } \\
\text { doença psiquiátrica ou TMM }\end{array}$ & & & & 0,004 \\
\hline Sim & 121 & 34,2 & 43,8 & \\
\hline Não & 232 & 65,5 & 28,4 & \\
\hline
\end{tabular}

Foi aplicado o teste de qui-quadrado.
Não foram relacionadas aos TMM as seguintes variáveis ( $p>0,05)$ : sexo masculino ou feminino, cor, possuir renda própria, renda familiar, ter uma religião específica ou ser membro praticante de alguma religião, morar com a família, utilizar medicação psiquiátrica, fazer psicoterapia e realizar automedicação (Tabela 2).

TABELA 2

Variáveis pesquisadas sem associação estatística com transtornos mentais menores (TMM) entre estudantes de Medicina da UFPB, $2012(\mathrm{~N}=354)$

\begin{tabular}{|c|c|c|c|c|}
\hline Variáveis & $\mathbf{N}$ & $\%$ & $\%$ TMM & $\mathbf{P}$ \\
\hline Sexo & & & & 0,277 \\
\hline Feminino & 175 & 49,4 & 30,9 & \\
\hline Masculino & 179 & 50,6 & 36,3 & \\
\hline Possui renda própria & & & & 0,268 \\
\hline Sim & 39 & 11 & 25,6 & \\
\hline Não & 307 & 86,7 & 34,5 & \\
\hline Renda familiar & & & & 0,46 \\
\hline Até R\$ 1.500 & 27 & 7,6 & 44,4 & \\
\hline Entre $R \$ 1.500$ e $R \$ 10.000$ & 209 & 59 & 32,5 & \\
\hline Acima de R\$ 10.000 & 91 & 25,7 & 35,2 & \\
\hline Cor & & & & 0,209 \\
\hline Branca & 193 & 54,5 & 31,6 & \\
\hline Parda & 138 & 39 & 38,4 & \\
\hline Preta & 13 & 3,7 & 23,1 & \\
\hline Amarela & 4 & 1,1 & 0 & \\
\hline Indígena & 3 & 0,8 & 66,7 & \\
\hline Religião & & & & 0,9 \\
\hline Católica & 166 & 46,9 & 30,7 & \\
\hline Evangélica & 25 & 7 & 28 & \\
\hline Espírita & 16 & 4,5 & 31,2 & \\
\hline Religioso praticante & & & & 0,956 \\
\hline Sim & 151 & 42,65 & 33,8 & \\
\hline Não & 203 & 57,3 & 33,5 & \\
\hline Mora com a família & & & & 0,098 \\
\hline Sim & 206 & 58,2 & 30,1 & \\
\hline Não & 148 & 41,8 & 38,5 & \\
\hline $\begin{array}{l}\text { Já fez ou faz tratamento } \\
\text { psiquiátrico medicamentoso }\end{array}$ & & & & 0,379 \\
\hline Sim & 40 & 11,3 & 40 & \\
\hline Não & 312 & 88,1 & 33 & \\
\hline $\begin{array}{l}\text { Já fez ou faz tratamento } \\
\text { psicoterapêutico }\end{array}$ & & & & 0,074 \\
\hline Sim & 62 & 17,5 & 43,5 & \\
\hline Não & 290 & 81,9 & 31,7 & \\
\hline Faz uso de automedicação & & & & 0,104 \\
\hline Sim & 122 & 34,5 & 39,3 & \\
\hline Não & 231 & 65,25 & 30,7 & \\
\hline
\end{tabular}

Foi aplicado o teste de qui-quadrado.

\section{DISCUSSÃO}

Embora as taxas de prevalência de TMM identificadas entre os estudantes de Medicina sejam extremamente variáveis, a 
prevalência encontrada neste estudo $(33,6 \%)$ foi comparável à de outros estudos brasileiros, permanecendo dentro do intervalo de valores já apresentados. A prevalência foi superior às encontradas na Universidade Federal de Santa Maria (31,7\%), na Universidade Federal do Rio Grande do Sul (22,19\%) e na Universidade Federal da Bahia $(29,6 \%)^{16,11,17}$, mas inferior às encontradas na Universidade Federal de Pernambuco (42,6\%) e na Universidade Estadual Paulista em Botucatu $(44,7 \%)^{12,13}$.

Este estudo apontou maior prevalência de TMM entre os alunos do curso básico. Após o entusiasmo inicial da conquista de uma vaga no curso mais disputado no vestibular e a entrada na universidade, os alunos se deparam com uma fase de frustração, causada pela mudança de hábitos do cotidiano, dificuldade na administração do tempo entre uma excessiva carga de estudos e pouco tempo para atividades de lazer ${ }^{18}$.

Nos resultados estratificados por período do curso, a maior frequência foi encontrada no quinto período, que é um momento de transição para os estágios clínicos do curso, com disciplinas como Gastroenterologia e Pneumologia. Não há consenso na literatura sobre o momento do curso em que o risco de desenvolver transtornos mentais é maior, pois esse dado sofre influência das características de cada escola médica, das disciplinas, dos professores e dos alunos envolvidos, o que torna complexa a comparação com outros estudos ${ }^{16,19,13}$. No internato, mesmo com a proximidade do fim da graduação, das provas de residência e da inserção no mercado de trabalho, encontrou-se a menor frequência de casos suspeitos de TMM de todo o curso ${ }^{1}$, por ser um grupo com faixa etária com mais de 20 anos, fase adulta jovem, com a personalidade já formada e com maior amadurecimento, capacitando-os a lidar melhor com os estressores.

A idade, assim como no estudo de Benvegnú et al. (1996) ${ }^{11}$, se associou a ser "suspeito" de TMM, com maior prevalência entre os estudantes de até 19 anos (42,6\%), por ser um grupo que se encontra na adolescência e por ter que lidar, além do curso, com as transformações e conflitos inerentes desse período.

Não houve associação entre as condições sociodemográficas (cor e renda familiar) dos estudantes de Medicina e TMM. A explicação possível é que, por se tratar de um grupo muito homogêneo em suas características sociodemográficas e econômicas, as pequenas diferenças encontradas perderam importância diante de outras categorias mais discrepantes no grupo $^{13}$.

Há relatos na literatura de associação entre sexo feminino e casos suspeitos de TMM na população geral ${ }^{20}$, porém sexo não foi uma variável estatisticamente significativa neste estudo, como também ocorreu em outros estudos entre estudantes de Medicina ${ }^{16,12,13}$.
Não foram relacionados ao TMM morar com a família e exercer atividade remunerada, ao contrário do que foi demonstrado no estudo de Giglio $^{10}$, pois morar com a família deveria estar associado com menores taxas de TMM, devido à rede de apoio que seria oferecida, e exercer atividade remunerada deveria estar ligado a maiores taxas de TMM, visto que o estudante divide seu tempo com outras atividades estressantes, além do curso. A automedicação, utilizada por muitos estudantes, por já se sentirem aptos ao próprio diagnóstico e tratamento, também não pôde ser correlacionada com o TMM.

$\mathrm{O}$ modelo multicausal de transtornos mentais prevê a influência de fatores genéticos e ambientais. Neste modelo, uma relação longitudinal causal entre os eventos estressores, o surgimento de sintomas e de transtornos mentais apresenta plausibilidade biológica aceitável. Segundo Kendler at al. ${ }^{21}$, numerosos estudos têm demonstrado que a exposição a eventos de vida estressores é substancialmente influenciada por fatores genéticos. Alguns indivíduos não se expõem a eventos de vida estressores ao acaso, mas apresentam tendência a selecionar situações com maior probabilidade de constituírem um evento de vida estressor. A avaliação negativa do evento estressor pode ser atribuída a uma vulnerabilidade que não está ligada ao evento estressor propriamente dito. A variabilidade individual provavelmente se deve a uma suscetibilidade mediada geneticamente, que influencia a forma de o indivíduo avaliar e enfrentar os eventos de vida estressores dependentes e independentes ${ }^{22}$. No presente estudo houve forte associação entre TMM e história familiar de transtorno mental, corroborando a influência dos fatores genéticos e ambientais.

Aqueles que seguem alguma religião, não importa qual seja, e mesmo os não religiosos praticantes tiveram menor incidência de TMM. A influência da espiritualidade na saúde física, mental e social tem sido amplamente demonstrada ${ }^{23,24,25}$. Há evidências crescentes de que a religiosidade - uma das dimensões da espiritualidade - está associada com saúde mental. Um estudo de revisão mostrou associação positiva com a religiosidade em $50 \%$ dos casos e negativa em $25 \%$ deles. Nessa revisão, a religiosidade foi considerada um fator protetor para suicídio, abuso de drogas e álcool, comportamento delinquente, satisfação marital, sofrimento psicológico e alguns diagnósticos de psicoses funcionais ${ }^{26}$.

Estudos transversais têm como limitação a impossibilidade de atribuir causalidade ou consequência às associações encontradas, já que analisam desfecho e exposição simultaneamente. Entretanto, apontam as direções nas quais os fatores preditivos se associam com o desfecho estudado. Procurou-se minimizar o viés de informação com o anonimato das respostas e a utilização de instrumentos autoaplicáveis, apenas com 
respostas objetivas. Quanto a erros na classificação do TMM, buscou-se um instrumento validado anteriormente no Brasil ${ }^{27}$, escolhendo-se um ponto de corte baseado em estudos anteriores com estudantes de Medicina ${ }^{12,13}$. É importante ressaltar, porém, que a avaliação pode ter sido influenciada pelo momento vivido por cada estudante na ocasião, já que o questionário deveria ser respondido com base nos 30 dias anteriores. Da mesma forma, os casos suspeitos de TMM podem não ter sido detectados nos estudantes já em acompanhamento psiquiátrico ou psicológico ${ }^{1}$

Deve-se considerar que o SRQ-20 simplesmente rastreia casos suspeitos de TMM, sendo o padrão-ouro para diagnóstico a entrevista com o psiquiatra ${ }^{28}$. Estudos longitudinais sobre morbidade psiquiátrica seriam mais adequados para estimar a ocorrência de transtornos mentais durante a vida acadêmica e a influência do currículo médico nesse contexto, pois poderiam contribuir para a identificação de fatores de risco potenciais no desenvolvimento de TMM nessa população ${ }^{12}$.

Diversos são os fatores preditivos para TMM relatados na literatura. Sentir-se sobrecarregado, presença de situações especiais durante a infância e adolescência que indiquem sofrimento mental preexistente, alterações no padrão de sono, avaliação ruim sobre desempenho escolar, dificuldade de fazer amigos, pensar em abandonar o curso e não receber o apoio emocional que necessita foram identificados na literatura como alguns dos fatores de maior risco para a ocorrência de TMM $^{1,16,12,13,29,30}$

As instituições de ensino superior devem refletir criticamente sobre o contexto do ensino médico, conhecer as características de seus alunos e os momentos considerados cruciais ao longo do curso, com a finalidade de articular estratégias para auxiliar o estudante a enfrentar as dificuldades do cotidiano ${ }^{13}$. A tarefa primordial é fornecer ao aluno um espaço para reflexão sobre seus sentimentos e emoções, mediante debate aberto e franco sobre suas vulnerabilidades, limitações e patologias $^{31}$.

Dados sobre TMM são importantes para subsidiar ações de prevenção e cuidado com a saúde mental da população, melhorando a qualidade de vida e, consequentemente, auxiliando em sua formação profissional.

Estudantes universitários são considerados um grupo especial de investimento social do País, particularmente em razão de funções de liderança que deverão exercer na sociedade em futuro próximo ${ }^{32}$. Estudos devem ser empreendidos dando ênfase às dimensões mais vulneráveis nessa fase da vida.

Sugere-se que as instituições formadoras estejam atentas, estabelecendo intervenções voltadas ao acolhimento e ao cuidado em relação ao sofrimento dos estudantes.

\section{CONCLUSÃO}

Os resultados encontrados demonstram que, nessa população, existe alta prevalência de TMM e que o período do curso, idade, religiosidade, história familiar de TMM ou doenças psiquiátricas e as queixas psicossociais são os fatores mais associados aos quadros desses transtornos. A alta prevalência encontrada pode estar associada a fatores presentes desde antes da graduação, o que ficou demonstrado pelo maior acometimento dos estudantes do início do curso, fato que pode estar relacionado até mesmo aos motivos que levam esses alunos a escolherem a carreira médica.

Essas informações são importantes para subsidiar ações de prevenção e cuidado com a saúde mental dos estudantes de Medicina, melhorando a qualidade de vida deles e auxiliando em sua formação profissional ${ }^{1}$.

\section{REFERÊNCIAS}

1. Fiorotti KP, Rossoni RR, Borges LH, Miranda AE. Transtornos mentais comuns entre os estudantes do curso de medicina: prevalência e fatores associados. J Bras Psiquiatr. 2010;59(1):17-23.

2. Coutinho ESF, Almeida Filho N, Mari JJ. Fatores de risco para morbidade psiquiátrica menor: resultados de um estudo transversal em três áreas urbanas no Brasil. Rev Psiquiatr Clín. 1999;26(5):246-56.

3. Cerchiari EAN. Saúde mental e qualidade de vida em estudantes universitários. Campinas; 2004. Doutorado [Tese] - Universidade Estadual de Campinas, Faculdade de Ciências Médicas.

4. Mowbray CT, Megivern D, Mandiberg JM, Strauss S, Stein $\mathrm{CH}$, Collins K, et al. Campus Mental Health Services: Recommendations for Change. Americ J Orthops. 2006;76(2):226-237.

5. Fernandez JM, Rodrigues CRC. Estudo retrospectivo de uma população de estudantes de medicina atendidos no ambulatório de Clínica Psiquiátrica do Hospital das Clínicas da Faculdade de Medicina de Ribeirão Preto. Med Ribr Pret. 1993;26(1):258-69.

6. Adlaf EM. The prevalence of elevated psychological distress among Canadian undergraduates: findings from the 1998 Canadian Campus Survey. J Americ Colleg Heal. 2001;50(2):67-72.

7. Kessler RC, Chiu WT, Demler O, Merikangas KR, Walters EE. Prevalence, severity, and comorbidity of 12-month DSM-IV disorders in the National Comorbidity Survey replication. Arch Gen Psyciquiatr. 2005;62(6):617-27.

8. Lépine JP. Prévalence et comorbidité des troubles psychiatriques dans la population générale française. L'Encéph. 2005;31(4):182-94. 
9. Loreto G. Sobre problemas de higiene mental. Neurob. 1958;21(3/4):274-83.

10. Giglio JS. Bem-estar emocional em estudantes universitários. Campinas; 1976. Doutorado [Tese] — FCM/UNICAMP.

11. Benvegnú LA, Deitos F, Copette FR. Problemas psiquiátricos menores em estudantes de medicina da Universidade Federal de Santa Maria, RS, Brasil. Rev Psiquiatr Rio Gd Sul. 1996;18(1):229-33.

12. Facundes VLD, Ludermir AB. Transtornos mentais comuns em estudantes da área de saúde. Rev Bras Psiquiatr. 2005;27(3):194-200.

13. Lima MCP, Domingues MS, Cerqueira ATAR. Prevalência e fatores de risco para transtornos mentais comuns entre estudantes de medicina. Rev Saúde Pública. 2006;40(6):1035-41.

14. Romaro RA, Capitão CG. Caracterização da clientela da clínica-escola de psicologia da Universidade São Francisco. Rev Pisicol Teoria e Prática. 2003;5(1):111-21.

15. Harding TW. Mental disorders in primary health care: a study of their frequency and diagnosis in four developing countries. Psycholog Med, 1980; 10(2)231-241.

16. Almeida AM, Godinho TM, Bitencourt AG, et al. Common mental disorders among medical students. J Bras Psiquiatr. 2007;56(4):245-51.

17. Loayza HMP, Ponte TS, Carvalho CG, Pedrotti MR, Nunes PV, Souza CM, et al. Association between mental health screening by self-report questionnaire and insomnia in medical students. Arq Neuropsiquiatr. 2001;59(2A):180-5.

18. Rezende CHA, Abrão CB, Coelho EP, Passos LBS. Prevalência de sintomas depressivos entre estudantes de medicina da Universidade Federal de Uberlandia. Rev Bras Educ Med. 2008;32(3):315-23.

19. Rosal MC, Ockene IS, Ockene JK, Barret SV, Ma Y, Hebert JR. A longitudinal study of students depression at one medical school. Acad Med. 1997;72(1):542-6.

20. Organizacão Mundial da Saude. Relatório mundial da saúde. Saúde mental: nova concepção, nova esperança. Lisboa; 2001.

21. Kendler KS, Karkowski LM, Prescott CA. Causal relationship between stressful life events and the onset of major depression. Am J Psychiatry. 1999;156(1):837-41.

22. Margis R. Relação entre estressores, estresse e ansiedade. Rev Psiquiatr. 2003;25(1):65-74.

23. World Health Organization. Division of mental health and prevention of substance abuse. WHOQOL and spirituality, religiousness and personal beliefs (SRPB) - report on WHO Consultation. Geneve: WHO; 1998.
24. Lotufo Neto F. Psiquiatria e religião: a prevalência de transtornos mentais entre ministros religiosos. São Paulo; 1997. Livre Docência [Tese] — Universidade Federal de São Paulo.

25. Sousa PLR, Tillmann IA, Horta CL, Oliveira FM. A religiosidade e suas interfaces com a medicina, a psicologia e a educação: o estado de arte. Psiq Prat Med. 2001;34(4):112-7.

26. Levin, JS, Chatters LM. Research on religion and mental health: an overview of empirical findings and theoretical issues. Koenig HG, ed. Handbook of religion and mental health. London: Academic Press; 1998. p.33-50.

27. Mari JJ, Williams PA. A validity study of a Psychiatric Screening Questionnaire (SRQ 20) in primary care in city of Sao Paulo. Br J Psychiatry. 1986;148(1):23-26.

28. Costa AG, Ludermir AB. Transtornos mentais comuns e apoio social: estudo em comunidade rural da Zona da Mata de Pernambuco, Brasil. Cad Saúde Pública. 2005;21(1):73-9.

29. Johnson WDK. Predisposition to emotional distress and psychiatric illness amongst doctors: the role of unconscious and experimental factors. Br J Med Psychol. 1991;64(1):317-29.

30. Ludermir AB, Melo Filho DA. Condições de vida e estrutura ocupacional associadas a transtornos mentais comuns. Rev Saúde Pública. 2002;36(2):213-21.

31. Millan LR, Arruda PCV. Assistência psicológica ao estudante de medicina: 21 anos de experiência. Rev Assoc Med Bras. 2008;54(1):90-4.

32. Andrade AG de, Bassit AZ, Kerr-Corrêa F, Tonhon AA, Boscovitz EP, Cabral M, et al. Fatores de risco associados ao uso de álcool e drogas na vida, entre estudantes de medicina do estado de São Paulo. Rev ABP-APAL. 1997;19(4):117-26.

\section{CONTRIBUIÇÃO DOS AUTORES}

Emmanuelle Santana Rocha pesquisou, avaliou os dados e escreveu o artigo. André Petraglia Sassi escreveu o artigo

\section{CONFLITO DE INTERESSES}

Emmanuelle Santana Rocha é aluna da instituição pesquisada e André Petraglia Sassi é funcionário da mesma instituição, Universidade Federal da Paraíba.

\section{ENDEREÇO PARA CORRESPONDÊNCIA}

Emmanuelle Santana Rocha

Rua Nurisman de Andrade Carneiro, 255

Bancários - João Pessoa

CEP. 58052-284 PB

E-mail: emmanuelle_sr@hotmail.com 